\title{
Influence of complete administration of adjuvant chemotherapy cycles on overall and disease-free survival in locally advanced rectal cancer: post hoc analysis of a randomized, multicenter, non- inferiority, phase 3 trial
}

Flavius Sandra-Petrescu ${ }^{1 *}$ (D) Florian Herrle ${ }^{1}$, Iris Burkholder ${ }^{2}$, Peter Kienle ${ }^{1}$ and Ralf-Dieter Hofheinz ${ }^{3}$

\begin{abstract}
Background: A randomized trial demonstrated that capecitabine is at least as effective as fluorouracil in the adjuvant treatment of patients with locally advanced rectal cancer. However, not all patients receive all planned cycles of chemotherapy. Therefore it is of interest how complete or partial administration of chemotherapy influences oncological outcome.

Methods: A post hoc analysis of a trial with 401 randomized patients, nine being excluded because of missing data, was performed. 392 patients (197 - capecitabine, 195 - fluorouracil) could be analyzed regarding the number of administered adjuvant chemotherapy cycles. In the subgroup of 361 patients with an overall survival of at least six months, five-year overall and disease-free survival were analyzed in respect to completion (complete vs. incomplete) of chemotherapy cycles. Survival rates and curves were calculated and compared using the log-rank test. The effect of completion of chemotherapy was adjusted for relevant confounding factors.

Results: Two hundred fifty-one (64.0\%) of analyzed patients received all postoperative scheduled cycles. Fiveyear overall survival was significantly better in these patients compared to the incomplete group (76.0 vs. 60 . $6 \%, p<0.0001)$. Of 361 patients with an overall survival of at least six months, 251(69.5\%) patients received all cycles. Five-year overall survival was also significantly better than in the incomplete group (76.0 vs. $66.4 \%, p=$ 0.0073). Five-year disease free survival was numerically better ( 64.9 vs. $58.7 \%, p=0.0646$; HR [not all cycles vs. all cycles $]=1.42$ 95\% Cl: $[0.98,2.07])$. Cox regression models show a non-significant better OS $(p=0.061)$ and DFS $(p=0.083)$, if chemotherapy cycles were administered completely.
\end{abstract}

Conclusion: Complete administration of chemotherapy cycles was associated with improved five-year overall and disease-free survival in patients with locally advanced rectal cancer.

Keywords: Completeness of chemotherapy, Rectal cancer, Overall survival, Disease-free survival, Curative resection

\footnotetext{
* Correspondence: flavius.sandra-petrescu@umm.de

'Surgical Department, University Medical Centre Mannheim,

Theodor-Kutzer-Ufer 1-3, 68167 Mannheim, Germany

Full list of author information is available at the end of the article
} 


\section{Background}

Multimodal treatment of locally advanced rectal cancer has continuously improved, resulting in better oncological outcome of affected patients. Current standard includes optimized surgery as defined by low anterior resection and total mesorectal excision (LAR and TME), combined with neoadjuvant (chemo)radiotherapy (long-term or short-course radiotherapy) and adjuvant chemotherapy. Locoregional recurrences have thus been considerably reduced but distant metastases are still the main problem. Several modifications of the standard, individual- or riskadapted, are currently being investigated in order to further improve prognosis in rectal cancer. Optimized and patienttailored neoadjuvant chemoradiotherapy (CRT) concepts with the aim of reducing local and distant recurrences are currently being tested in clinical trials [1]. The use of magnetic resonance imaging (MRI) predicting the circumferential resection margin (CRM) is now widely used in order to avoid neoadjuvant CRT in low risk cases [2, 3]. Also the role of adjuvant chemotherapy in patients with locally advanced rectal cancer undergoing neoadjuvant CRT and TME is at this time a matter of debate [4]. Moreover, omission of both, surgery and adjuvant chemotherapy is evaluated in selected patients with a complete response achieved with neoadjuvant CRT [5].

However, according to German and other international guidelines, neoadjuvant CRT and fluorouracil based adjuvant chemotherapy remain a standard of care in the multimodal treatment of stage II - III rectal cancers (cT3-4 N0 M0, cT any N1-2 M0:T1 - tumour invades submucosa, T2 - tumour invades muscularis propria, T3 - tumour invades trough the muscularis propria into pericolorectal tissues, T4 - tumour directly invades other organs or structures and/or perforates visceral peritoneum, N0 - no regional lymph node metastasis, N1 metastasis in 1 to 3 regional lymph nodes, N2 - metastasis in 4 or more regional lymph nodes, M0 - no distant metastasis; $\mathrm{c}$ - clinical, $\mathrm{p}$ - pathologic; as defined by the AJCC7ed) [6-8]. As capecitabine, as an adjuvant treatment regime, was shown to be non-inferior to 5-FU regarding relapse-free survival in stage III ( $\mathrm{cT}_{\text {any }} \mathrm{N} 1-2 \mathrm{M} 0$ ) colon cancer it was also investigated as perioperative treatment in locally advanced rectal cancer [8-10]. A phase 3 multicenter trial demonstrated that capecitabine was at least as effective as fluorouracil in the neoadjuvant CRT and adjuvant setting within a multimodal treatment concept for patients with stage II-III (c or pT3-4 $\mathrm{N}_{\text {any }}$, or c or $\mathrm{pT}_{\text {any }} \mathrm{N}_{\text {positive, }}$, M0) rectal cancer [8]. Overall survival (OS) was noninferior in the capecitabine compared with the fluorouracil group. Interestingly disease-free survival (DFS) was proven to be better in the capecitabine group due to fewer distant metastases [10]. Therefore, adjuvant treatment obviously plays a role in controlling distant metastases and in this context it seems sensible to administer all planned adjuvant chemotherapy cycles in order to potentially maximize the oncological benefit. But not all patients indeed receive all planed chemotherapy cycles and there is scarce data on whether completeness of chemotherapy cycles $(\mathrm{CoC})$ really has a relevant effect on oncological outcome and if so how large this effect is. In order to determine whether CoC influences five-year OS and DFS a post hoc reanalysis of the data from our previous phase-III study was performed [10].

\section{Methods}

A detailed description of the trial including inclusion and exclusion criteria as well as randomization, statistical aspects and other eligibility criteria was previously given [10]. Shortly this was a two-arm, two-cohort, multicentre, randomized, open-label, non-inferiority, phase 3 trial comparing fluorouracil with capecitabine for perioperative treatment of patients with locally advanced rectal cancer. Initially designed as an adjuvant trial the protocol was amended to allow a neoadjuvant cohort. The trial protocol was approved by the institutional review boards of all participating centres. All participants provided written informed consent. Newly diagnosed patients with locally advanced rectal adenocarcinoma, stage II - III (pT3-4 $\mathrm{N}_{\text {any }}$ or $\mathrm{pT}_{\text {any }} \mathrm{N}_{\text {positive }}, \mathrm{M} 0$ for adjuvant cohort respectively cT3-4 $\mathrm{N}_{\text {any }}$ or $\mathrm{CT}_{\text {any }} \mathrm{N}_{\text {positive }}$, M0 for neoadjuvant cohort), with a distal tumour border $<16 \mathrm{~cm}$ from anal verge were recruited in 35 German institutions between March, 2002, and December, 2007 [8]. All patients were resected in curative intent as confirmed by the histological results (R0). A partial or total mesorectal excision (PME or TME) was performed for tumours localized in the upper third and lower two-thirds of rectum, respectively.

Patients were randomized into two groups, capecitabine or fluorouracil, in a 1:1 ratio using permuted blocks, with stratification by centre and pathological tumour stage. In the adjuvant cohort the capecitabine group was scheduled to receive two cycles of capecitabine $\left(2500 \mathrm{mg} / \mathrm{m}^{2}\right.$ days $1-$ 14, repeated day 22), followed by CRT (50.4 Gy plus capecitabine $1650 \mathrm{mg} / \mathrm{m}^{2}$ days 1-38), then three cycles of capecitabine. Patients randomized into the neoadjuvant cohort were planned to receive CRT (50.4 Gy plus capecitabine $1650 \mathrm{mg} / \mathrm{m}^{2}$ daily) followed by radical surgery and afterwards five cycles of capecitabine $\left(2500 \mathrm{mg} / \mathrm{m}^{2}\right.$ per day for 14 days).

In the adjuvant cohort the planned therapy for patients receiving fluorouracil included two cycles of bolus fluorouracil (500 $\mathrm{mg} / \mathrm{m}^{2}$ days $1-5$, repeated day 29), followed by CRT (50.4 Gy plus infusional fluorouracil $225 \mathrm{mg} / \mathrm{m}^{2}$ daily), then two cycles of bolus fluorouracil. Patients in the neoadjuvant cohort received CRT (50. 4 Gy plus infusional fluorouracil $1000 \mathrm{mg} / \mathrm{m}^{2}$ days $1-5$ and 29-33) followed by radical surgery and afterwards four cycles of bolus fluorouracil ( $500 \mathrm{mg} / \mathrm{m}^{2}$ for 5 days). 
The overall population of the study consisted of 392 patients. A further subgroup of 361 patients with an overall survival time of at least 6 months was analyzed. Comparisons were performed between patients receiving all (complete $[\mathrm{CoC}]$ - group) scheduled therapy cycles (5-FU arm: 5 cycles, capecitabine arm: 6 cycles) versus all patients receiving not all scheduled cycles (incomplete $[$ non-CoC] - group $=$ at least one cycle less of the planned chemotherapy).

\section{Statistical methods}

Baseline characteristics were analyzed using descriptive statistics. OS was calculated from the date of randomization to the date of death. DFS was calculated from the date of randomization to the date of disease recurrence (metastasis or local recurrence), development of a second primary cancer (including non-colorectal carcinoma), or death from any other cause, whichever occurred first. OS as well as DFS were analyzed using censored failure times with the Kaplan-Meier method. Kaplan-Meier curves for OS and DFS were compared using the log-rank test. Five-year survival rates as well as Cox proportional hazard rates (HR) and 95\% confidence intervals were calculated for OS and DFS. In addition, univariate Cox regression analysis was performed for completion of chemotherapy as well as for all baseline characteristics of the study population (age, gender, therapy arm, cohort, WHO status, tumor and nodal category). Cox proportional HRs as well as $95 \%$ confidence intervals were calculated. Global null hypothesis was tested using likelihood ratio test. Parameters which were significant in univariate analysis at a 5\% significance level were included in a multivariate Cox regression with the aim to adjust the effect of completion of chemotherapy for relevant confounding factors. Univariate as well as multivariate Cox regression was performed for OS and DFS. Data were analyzed using the statistical package SAS for Windows version 9.4 (SAS Institute Inc., North Carolina).

\section{Results}

Of 401 randomized patients nine were excluded because of missing post-randomization data. Therefore 392 patients were analyzed, 197 in the capecitabine and 195 in the fluorouracil group. Thereof, 231 patients were included in the adjuvant cohort and 161 in the neoadjuvant cohort. 251 (64\%) patients in both groups, capecitabine and fluorouracil, respectively completed the planned adjuvant chemotherapy cycles (6 cycles capecitabine and 5 cycles 5 -FU). CoC group $(N=251)$ and non-CoC group $(N=141)$ were compared by treatment and number of cycles. The pooled analysis of 5-FU and capecitabine $\mathrm{CoC}$ vs. non-CoC cycles showed a significantly better 5-year OS 76\% (95\% CI $69.1-81.6 \%$ ) in the CoC group vs. $60.6 \%$ (95\% CI 48. $0-71.0 \%, p<0.0001$ ) in the non-CoC group (Fig. 1).
A total of $N=31$ patients of the original population have OS lower than 6 months. Reasons for end of study of those patients are:

- Death (10 patients- among them 2 tumour related, 1 therapy related, 3 unrelated to tumour or therapy, 4 missing reasons)

- Withdrawal of consent (11 patients)

- Lost to follow-up (1 patient)

- Protocol deviation (3 patients)

- Toxicity (1 patient)

- Death and toxicity (1 patient)

- Others (4 patients).

Looking further at the subgroup of patients with at least 6 months OS (Table 1), 251 (69.53\%) of 361 patients, received all cycles (CoC) and $110(30.47 \%)$ patients received at least one cycle less (non-CoC) (Table 2). Most patients have a T3 tumour (clinical or pathological) and are node positive.

Looking further at the number of received cycles, 12 (33\%) of 36 patients (8 capecitabine and $45-\mathrm{FU})$ in the adjuvant cohort and $9(12 \%)$ of 74 patients (3 capecitabine and 35 -FU) in the neoadjuvant cohort received one cycle less than planned (Table 2). Overall, 19\% of patients in the non-CoC group received only one cycle less. Looking at the chemotherapy regimens within the non-CoC group, 11 (10\%) patients in the capecitabine group and $7(6 \%)$ patients in the 5 -FU group received one cycle less than the planned therapy.

The analysis of this subgroup of population showed a significantly better $\mathrm{OS}$ in the $\mathrm{CoC}$ group compared to the non-CoC group, 76.0\% (95\% CI 69.1\%, 81.6\%) vs. 66. $4 \%(52.7 \%, 76.9 \%), p=0.0073$; HR for non-CoC vs. CoC 1.84 95\% CI: [1.17, 2.90] (Fig. 2).

The pooled analysis of 5-year disease-free survival showed a non-significant better outcome in the CoC group $64.9 \%(95 \%$ CI $57.8-71.0 \%)$ compared to the non-CoC group 58.7\% (95\% CI46.7-68.8\%, $p=0.0646$ ); HR for non-CoC vs. CoC was 1.42 (95\% CI 0.98-2.07) (Fig. 3). Univariate as well as multivariate Cox regression analyses of OS and DFS by baseline characteristics for the study population are presented as Additional file 1.

\section{Discussions}

In our post hoc analysis complete administration of planned adjuvant chemotherapy (6 cycles capecitabine and 5 cycles 5 -FU) led to a better survival in patients with locally advanced rectal cancer. The Cox regression shows that even after adjustment $\mathrm{CoC}$ still leads to a clinically relevant, yet not statistically significant better survival. When looking specifically at the different chemotherapy regimens but also at the adjuvant and neoadjuvant cohort most of the patients within the non-CoC group received 


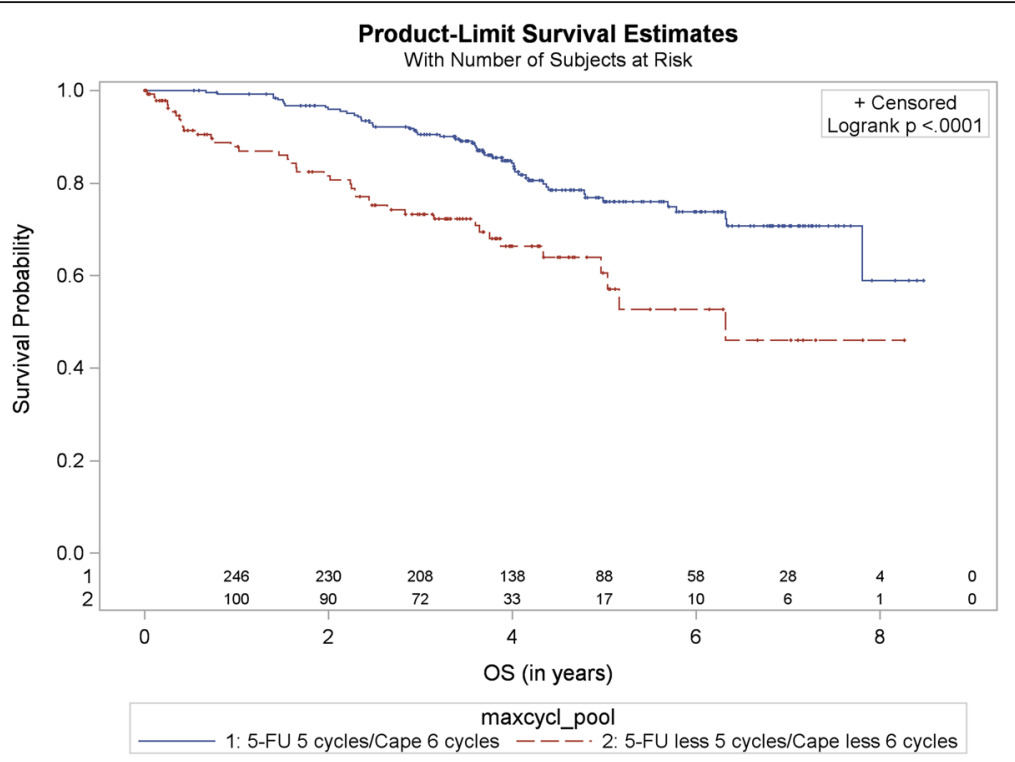

Fig. 1 Kaplan-Meier curve for overall survival (in years). Comparison by treatment and number of cycles. Pooled analysis of 5-FU vs. capecitabine, CoC (5-FU 5 cycles/capecitabine 6 cycles) vs. non-CoC (5-FU less 5 cycles/capecitabine less 6 cycles). Cape - capecitabine. 1and 2 - number at risk

less than only one cycle less than complete and this resulted in a worse oncological outcome. These results and others indicate that it is of importance that patients undergo all chemotherapy cycles in order to maximally benefit from adjuvant treatment [11]. A large randomized controlled trial comparing neoadjuvant to adjuvant treatment in rectal cancer demonstrated $\mathrm{CoC}$ in only $50.0 \%$ of patients [12]. This is similar to a $\mathrm{CoC}$ rate of $57.0 \%$ in a randomized study comparing induction chemotherapy prior to neoadjuvant chemo-radiotherapy [11]. In our study $\mathrm{CoC}$ pooled for both cohorts, adjuvant and neoadjuvant, capecitabine and 5-FU, was $64.0 \%$ if the entire population of the study was considered $(N=392)$ or 69 . $5 \%$ in case of the subgroup of patients with at least 6 months survival $(N=361)$. This is comparable to a recent retrospective analysis of 294 patients with rectal cancer, stage I-IV (IV - $\mathrm{T}_{\text {any }} \mathrm{N}_{\text {any }}$ M1: M1 - distant metastasis), undergoing neoadjuvant treatment, rectal resection with protective ileostomy and adjuvant therapy $[8,13]$. In this study $65 \%$ of the patients received a complete adjuvant therapy, which in contrast to our trial, was broadly defined as application of complete adjuvant chemotherapy for at least 3 months [13]. The multimodal therapy of locally advanced rectal cancer is continuously improving and neoadjuvant as well as adjuvant therapy strategies are currently, in the era of optimized surgery (TME), under discussion. Regarding neoadjuvant CRT several patient orientated concepts are developing [1]. These include organ preserving strategies, non-operative management, induction or consolidation chemotherapy and use of targeted agents $[1,5]$. The German and also most of the international guidelines still recommend adjuvant therapy of stage II - III rectal cancers, subsequent to neoadjuvant long course CRT or short-term radiotherapy $(5 \times 5$ Gy) and TME $[6,8,14]$. Contrarily, a recently published meta-analysis of four clinical trials concluded that adjuvant fluorouracil-based chemotherapy does not improve OS or DSF in rectal cancer patients with (y)pTNM stage II-III disease [4, 8]. These patients received adjuvant chemotherapy or underwent observation after (chemo)radiotherapy and surgery. We and others already commented the results of this analysis as the included studies all have considerable drawbacks in regard to their validity [15-17]. In the meta-analysis no data is provided regarding the influence of $\mathrm{CoC}$ and of dose intensity on overall survival which was the primary endpoint of the analysis. As also stated by the authors, there was poor compliance to adjuvant therapy since less than $50 \%$ of the patients received the planned dose $[4,15-18]$. No comparison between $\mathrm{CoC}$ and non-CoC was performed. This would be interesting since a complete (dose and cycles) administration of the chemotherapy might influence survival as now shown in our post hoc analysis. You et al. pointed out that it would be necessary, in order to analyze the effect of adjuvant chemotherapy on OS, to compare OS of patients who completely or incompletely received the adjuvant chemotherapy versus that of the observational group [16]. Patient selection in the studies included in the meta-analysis is somewhat unclear as a substantial number of patients from the I-CNR-RT and EORTC 2291 or those with an ypTNM0 and ypTNM1 tumour were excluded $[8,19,20]$. Moreover, a representative number of patients included into the analysis were from the PROCTOR-SCRIPT study which was closed 
Table 1 Baseline characteristics of the study population: patients with at least 6 months OS $(N=361)$

\begin{tabular}{|c|c|c|}
\hline & $\begin{array}{l}\text { CoC-Group } \\
(N=251)\end{array}$ & $\begin{array}{l}\text { non-CoC-Group } \\
(N=110)\end{array}$ \\
\hline $\begin{array}{l}\text { Median age, years (range; } \\
\text { interquartile range) }\end{array}$ & $63(30-83 ; 56.1-68.7)$ & $65(40-86 ; 56.4-71.5)$ \\
\hline \multicolumn{3}{|l|}{ Sex } \\
\hline Male & $164(65 \%)$ & 77 (70\%) \\
\hline Female & 87 (35\%) & $33(30 \%)$ \\
\hline \multicolumn{3}{|l|}{ WHO status } \\
\hline 0 & 139 (55\%) & $63(57 \%)$ \\
\hline 1 & 90 (36\%) & 40 (36\%) \\
\hline 2 & $2(1 \%)$ & $2(2 \%)$ \\
\hline Missing data & $20(8 \%)$ & $5(5 \%)$ \\
\hline \multicolumn{3}{|l|}{ Therapy arm } \\
\hline Capecitabine & 127 (51\%) & $53(48 \%)$ \\
\hline $5-\mathrm{FU}$ & 124 (49\%) & $57(52 \%)$ \\
\hline \multicolumn{3}{|l|}{ Cohort } \\
\hline Adjuvant & $182(73 \%)$ & $36(33 \%)$ \\
\hline Neoadjuvant & $69(27 \%)$ & $74(67 \%)$ \\
\hline \multicolumn{3}{|l|}{ Tumour category ${ }^{a}$} \\
\hline $\mathrm{T} 1$ or $\mathrm{T} 2$ & 48 (19\%) & $12(11 \%)$ \\
\hline T3 & 184 (73\%) & 89 (81\%) \\
\hline $\mathrm{T} 4$ & $18(7 \%)$ & $8(7 \%)$ \\
\hline Missing data & $1(<1 \%)$ & $1(1 \%)$ \\
\hline \multicolumn{3}{|l|}{ Nodal category ${ }^{a}$} \\
\hline Node negative & 93 (37\%) & 45 (41\%) \\
\hline Node positive & $157(63 \%)$ & $60(54 \%)$ \\
\hline Missing data & $1(<1 \%)$ & $5(5 \%)$ \\
\hline
\end{tabular}

Data are $\mathrm{n}(\%)$ or median (range). ${ }^{\mathrm{a}}$ clinical or pathological category. WHO World Health Organization, FU fluorouracil. T - size or direct extent of the primary tumor

prematurely and where a potential selection bias is evident [15]. Also patients with non-standard treatments such as 45Gy radiation without chemotherapy were included. Petrelli et al. argues that adjuvant chemotherapy should be especially considered in patients with locally advanced disease who experienced downstaging after neoadjuvant (chemo)radiotherapy [18]. But the patients included into the above cited meta-analysis were most probably mostly non-responders to neoadjuvant (chemo)radiotherapy since no or only minimally downstaging was achieved in this study.

In a pooled analysis of 11 studies Maas et al. showed that adjuvant therapy in rectal cancer may possibly be stratified on the basis of pathologic complete response (pCR) [21]. The authors found that after neoadjuvant CRT and TME ypT1-2 tumors benefited more from adjuvant treatment than ypT3-4 tumors $[8,21]$. But also in this study the limited effect of chemotherapy is potentially due to poor adherence to adjuvant therapy.
Table 2 Patients receiving scheduled treatment per cycle

\begin{tabular}{lll}
\hline & Capecitabine & Fluorouracil \\
\hline Adjuvant Cohort $^{1}$ & & \\
1 & $107(98 \%)$ & $109(100 \%)$ \\
2 & $105(96 \%)$ & $106(97 \%)$ \\
3 & $102(94 \%)$ & $101(93 \%)$ \\
4 & $99(91 \%)$ & $96(88 \%)$ \\
5 & $98(90 \%)$ & $92(84 \%)$ \\
6 & $90(83 \%)$ & - \\
Neoadjuvant Cohort ${ }^{2}$ & & $71(99 \%)$ \\
1 & $66(93 \%)$ & $46(64 \%)$ \\
2 & $50(70 \%)$ & $38(53 \%)$ \\
3 & $46(65 \%)$ & $35(49 \%)$ \\
4 & $42(59 \%)$ & $32(44 \%)$ \\
5 & $40(56 \%)$ & - \\
6 & $37(52 \%)$ & \\
\hline Data are $n$ (\%) $n=109$ Capecitabine, $n=109$ Fluorouracil; & \\
$2 n=71$ Capecitabine, $n=72$ Fluorouracil &
\end{tabular}

There is evidence that different combinations of chemotherapy regimens may be more suitable in selected patient groups. Therefore, recent clinical studies investigated whether addition of oxaliplatin to the bolus fluorouracil and capecitabine is more effective in the perioperative treatment of locally advanced rectal cancer. In several of these studies (e.g. PETACC-6) neoadjuvant CRT showed high toxicity in the experimental arm (addition of oxaliplatin to standard capecitabine- or 5-FU-based regimen) [22]. This resulted in less compliance in the PETACC-6 experimental group when compared to the standard arm (approximately $77 \%$ of the patients started adjuvant chemotherapy, less in the oxaliplatin group and 69\% completed it in the standard capecitabine arm and only $57 \%$ in the oxaliplatin arm). In the CAO/ARO/AIO-04 study, grade 3-4 side-effects were slightly more common in the 5 -FU/oxaliplatin combination group ( 21 vs. $15 \%$ ), but this had no effect on the rate of applied full chemotherapy dose [23]. In the PETACC-6 study, the primary endpoint was not reached (DFS standard arm $74.5 \%$ and + oxaliplatin $73.9 \%, p=0.78$ ) whereas in the $\mathrm{CAO} / \mathrm{ARO} / \mathrm{AIO}-04$ study there was a significant improvement of 3-year-DFS in the 5 -FU + oxaliplatin group ( 75.9 vs. $71.2 \%, p=0.03$ ).

The Korean phase II study ADORE evaluated whether addition of oxaliplatin to adjuvant therapy is of advantage in "high risk" histology [24]. 95\% of patients completed planned chemotherapy cycles and 3-year-DFS was significantly better in patients receiving oxaliplatin (72 vs. $63 \%, p=0.03$ ), however restricted to patients with stage III disease $[8,24]$.

Although ADORE and CAO/ARO/AIO-04 trials showed a significant advantage of adjuvant chemotherapy regarding 


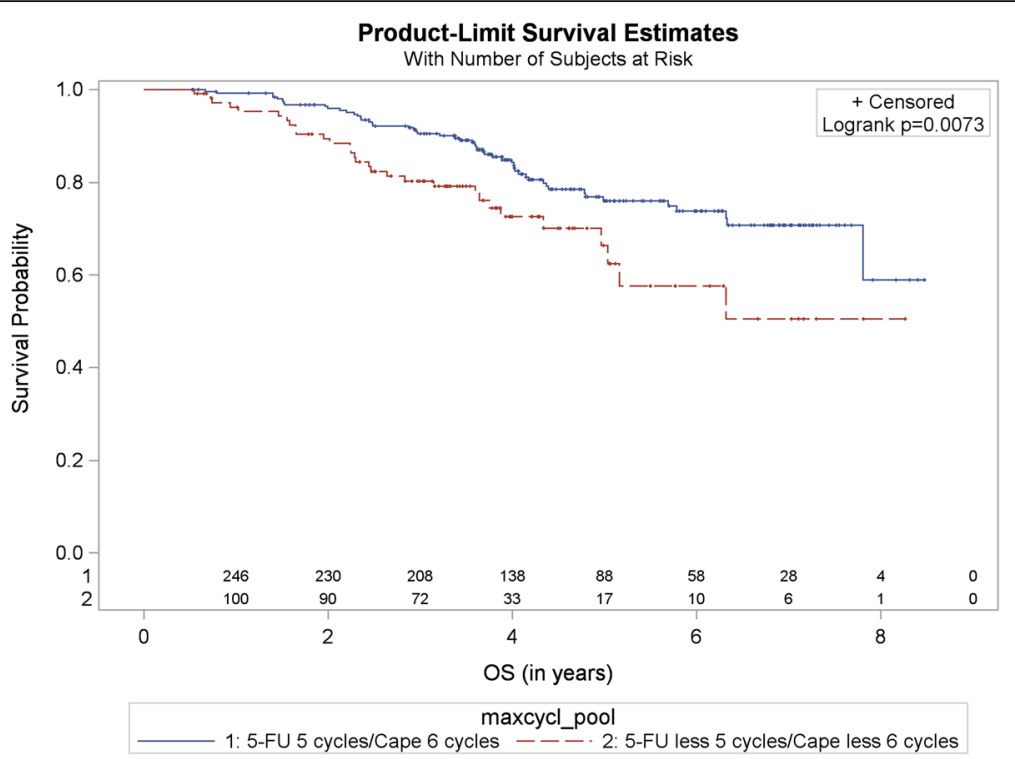

Fig. 2 Kaplan-Meier curve for overall survival in patients with at least 6 months survival (in years). Comparison by treatment and number of cycles. Pooled analysis of 5-FU vs. capecitabine, CoC (5-FU 5 cycles/capecitabine 6 cycles) vs. non-CoC (5-FU less 5 cycles/capecitabine less 6 cycles). Cape - capecitabine. 1 and 2 - number at risk

DFS when compared combined fluorouracil and oxaliplatin to fluorouracil alone, no further investigations were performed to determine whether complete vs. incomplete administration of the adjuvant chemotherapy significantly improve the OS or DFS [23, 24].

Our data show that $\mathrm{CoC}$ improves long-term oncological results, OS and DFS respectively, in rectal cancer patients when compared to incomplete adjuvant therapy.
In our analysis considerably more patients underwent all chemotherapy cycles in the adjuvant compared to the neoadjuvant group and this was similar in the capecitabine and 5-FU group. This is might be explained due to the additional side-effects of concomitant radiotherapy in the latter group. A higher rate of gastrointestinal sideeffects like proctitis and diarrhoea was noticed during radiotherapy in the capecitabine group [10]. Moreover,

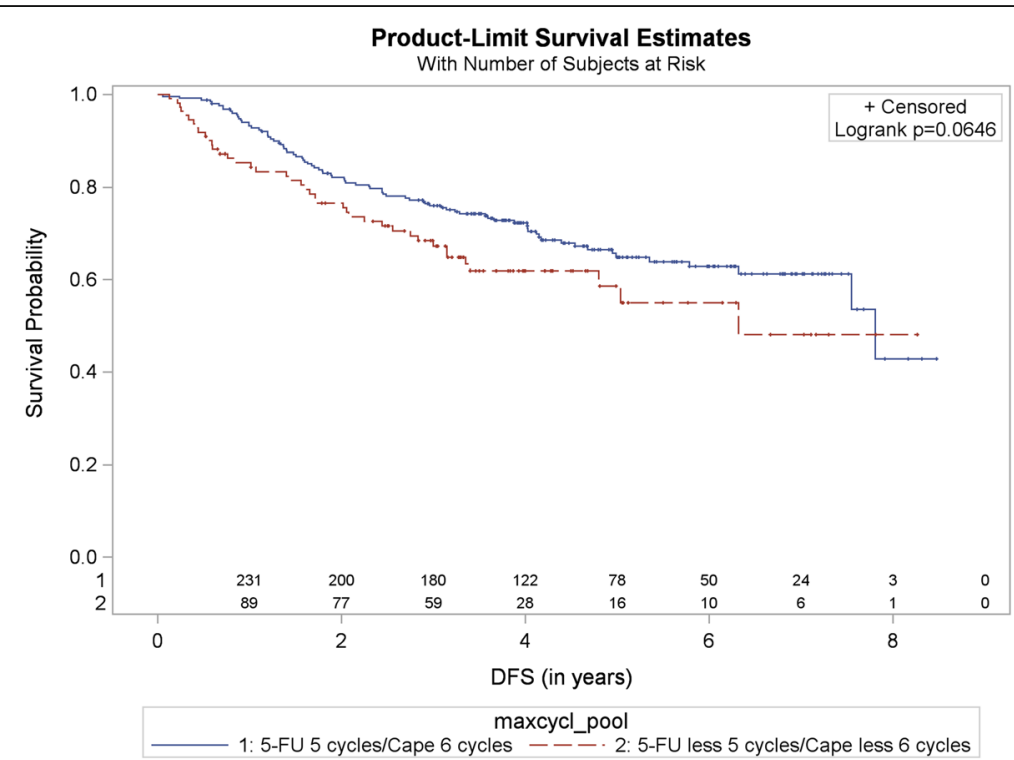

Fig. 3 Kaplan-Meier curve for disease free survival in patients with at least 6 months survival (in years). Comparison by treatment and number of cycles. Pooled analysis of 5-FU vs. capecitabine, CoC (5-FU 5 cycles/capecitabine 6 cycles) vs. non-CoC (5-FU less 5 cycles/capecitabine less 6 cycles). Cape - capecitabine. 1 and 2 - number at risk 
acute and chronic side-effects of neoadjuvant CRT or short course radiotherapy followed by surgery have been described to influence the postoperative course of the patients [25-29]. The toxicity of neoadjuvant therapy was shown to influence the rate of postoperative complications when compared to surgery alone [30]. A substantial percentage of patients will not be able to complete the scheduled adjuvant therapy. This is mostly due to suboptimal compliance with the therapy which can be explained by chemotherapy toxicity and postoperative complications such as stoma-related morbidity, high-output stoma, stoma prolapse or bowel occlusion, wound infection or anastomotic leakage [11, 31-34]. To improve $\mathrm{CoC}$ we designed a multicentre prospective randomized trial which addresses one of these factors which influence postoperative morbidity. This trial is analyzing the influence of a protective stoma on the administration of the complete adjuvant chemotherapy in patients with stage II - III rectal cancer $[8,35]$. $\mathrm{CoC}$ is the primary endpoint and the trial will allow us also to evaluate the influence of dose reduction on patients' survival. This trial is currently recruiting and 231 patients are randomized until now.

\section{Conclusions}

Complete administration of scheduled adjuvant chemotherapy cycles was associated with improved survival in patients with locally advanced rectal cancer. Patient compliance should be improved in order to increase the rate of completeness of chemotherapy.

\section{Additional file}

Additional file 1: Uni- and multivariate analyses for OS and DFS Univariate Cox regression models for $\mathrm{CoC}$ and baseline characteristics of the study population as well as adjustment of $\mathrm{CoC}$ effect for relevant confounding factors. (DOCX $45 \mathrm{~kb}$ )

\section{Abbreviations}

CoC: completeness of chemotherapy cycles; CRM: circumferential resection margin; CRT: chemoradiotherapy; DFS: disease - free survival; FU: fluorouracil; LAR: low anterior resection; MRI: magnetic resonance imaging; OS: overall survival; pCR: pathologic complete response; PME: partial mesorectal excision; TME: total mesorectal excision; Vs.: versus

\section{Acknowledgements}

Not applicable

\section{Funding}

This work has no specific funding.

\section{Availability of data and materials}

All data generated or analyzed during this study are included in this published article.

\section{Authors' contributions}

FS-P designed the study, analyzed the data and wrote the manuscript, $\mathrm{FH}$ analyzed the data and wrote the manuscript, IB performed the statistic, PK and R-DH designed the study and analyzed the data, wrote and supervised the work. All authors read and approved the final manuscript.

\section{Ethics approval and consent to participate}

The present study is a post hoc analysis of a randomized, multicentre, noninferiority, phase 3 trial which was already published (Hofheinz RD, Wenz F, Post S, et al. Chemoradiotherapy with capecitabine versus fluorouracil for locally advanced rectal cancer: a randomised, multicentre, non-inferiority, phase 3 trial. Lancet Oncol 2012; 13: 579-88.). The trial has been registered with ClinicalTrials.gov number NCT01500993. An ethical approval has been obtained for this trial from the 2nd Ethics Committee of the Medical Faculty of Mannheim, Germany. Therefore no supplementary ethical approval has been obtained for the present study.

\section{Consent for publication}

Not applicable

\section{Competing interests}

The authors declare that they have no competing interests

\section{Publisher's Note}

Springer Nature remains neutral with regard to jurisdictional claims in published maps and institutional affiliations.

\section{Author details}

${ }^{1}$ Surgical Department, University Medical Centre Mannheim, Theodor-Kutzer-Ufer 1-3, 68167 Mannheim, Germany. ${ }^{2}$ Department of Nursing and Health, University of Applied Sciences of the Saarland, Goebenstr. 40, D-66117 Saarbruecken, Germany. ${ }^{3}$ Day Treatment Center (TTZ), Interdisciplinary Tumor Center Mannheim (ITM) \& III Medical Clinic, Theodor-Kutzer-Ufer 1-3, 68167 Mannheim, Germany.

Received: 27 September 2017 Accepted: 26 March 2018

Published online: 03 April 2018

\section{References}

1. Rodel C, Hofheinz R, Fokas E. Rectal cancer: neoadjuvant chemoradiotherapy. Best Pract Res Clin Gastroenterol. 2016;30(4):629-39.

2. Sautter-Bihl ML, Hohenberger W, Fietkau R, Roedel C, Schmidberger H, Sauer R. MRI-based treatment of rectal cancer: is prognostication of the recurrence risk solid enough to render radiation redundant? Ann Surg Oncol. 2014;21(1):197-204

3. Kreis ME, Ruppert R, Ptok H, Strassburg J, Brosi P, Lewin A, Schon MR, Sauer $J$, Junginger $T$, Merkel $S$, et al. Use of preoperative magnetic resonance imaging to select patients with rectal Cancer for neoadjuvant Chemoradiation-interim analysis of the German OCUM trial (NCT01325649). Gastrointest Surg. 2016;20(1):25-32. discussion 32-23

4. Breugom AJ, Swets M, Bosset JF, Collette L, Sainato A, Cionini L, GlynneJones R, Counsell N, Bastiaannet E, van den Broek CB, et al. Adjuvant chemotherapy after preoperative (chemo)radiotherapy and surgery for patients with rectal cancer: a systematic review and meta-analysis of individual patient data. Lancet Oncol. 2015;16(2):200-7.

5. Habr-Gama A, Lynn PB, Jorge JM, Sao Juliao GP, Proscurshim I, GamaRodrigues J, Fernandez LM, Perez RO. Impact of organ-preserving strategies on anorectal function in patients with distal rectal Cancer following neoadjuvant Chemoradiation. Dis Colon rectum. 2016;59(4):264-9.

6. Pox C, Aretz S, Bischoff SC, Graeven U, Hass M, Heussner P et al. S3-guideline colorectal cancer version 1.1. 2014. [http://www.awmf.org/leitlinien/detail/II/ 021-007OL.html. Access 26 Sept 2017]

7. Lakkis Z, Manceau G, Bridoux V, Brouquet A, Kirzin S, Maggiori L, de Chaisemartin C, Lefevre JH, Panis Y, Snfcp TF. Management of rectal cancer: the 2016 French guidelines. Color Dis. 2016

8. American Joint Committee on Cancer. Colon and Rectum Cancer Satging. 7th edition. [https://cancerstaging.org/references-tools/quickreferences/ Documents/ColonMedium.pdf. Accessed 26 Sept 2017].

9. Twelves C, Wong A, Nowacki MP, Abt M, Burris H 3rd, Carrato A, Cassidy J, Cervantes A, Fagerberg J, Georgoulias V, et al. Capecitabine as adjuvant treatment for stage III colon cancer. N Engl J Med. 2005;352(26):2696-704.

10. Hofheinz RD, Wenz F, Post S, Matzdorff A, Laechelt S, Hartmann JT, Muller L, Link H, Moehler M, Kettner E, et al. Chemoradiotherapy with capecitabine versus fluorouracil for locally advanced rectal cancer: a randomised, multicentre, non-inferiority, phase 3 trial. Lancet Oncol. 2012;13(6):579-88.

11. Fernandez-Martos C, Pericay C, Aparicio J, Salud A, Safont M, Massuti B, Vera R, Escudero P, Maurel J, Marcuello E, et al. Phase II, randomized study of 
concomitant chemoradiotherapy followed by surgery and adjuvant capecitabine plus oxaliplatin (CAPOX) compared with induction CAPOX followed by concomitant chemoradiotherapy and surgery in magnetic resonance imaging-defined, locally advanced rectal cancer: Grupo cancer de recto 3 study. J Clin Oncol. 2010;28(5):859-65.

12. Sauer R, Becker H, Hohenberger W, Rodel C, Wittekind C, Fietkau R, Martus P, Tschmelitsch J, Hager E, Hess CF, et al. Preoperative versus postoperative chemoradiotherapy for rectal cancer. N Engl J Med. 2004;351(17):1731-40.

13. Phatak UR, Kao LS, You YN, Rodriguez-Bigas MA, Skibber JM, Feig BW, Nguyen S, Cantor SB, Chang GJ. Impact of ileostomy-related complications on the multidisciplinary treatment of rectal cancer. Ann Surg Oncol. 2014;21(2):507-12.

14. van de Velde CJ, Boelens PG, Borras JM, Coebergh JW, Cervantes A Blomqvist L, Beets-Tan RG, van den Broek CB, Brown G, Van Cutsem E, et al. EURECCA colorectal: multidisciplinary management: European consensus conference colon \& rectum. Eur J Cancer. 2014;50(1):1 e1-1 e34.

15. Hofheinz RD, Rodel C, Burkholder I, Kienle P. Adjuvant chemotherapy for rectal cancer. Lancet Oncol. 2015;16(4):e154-5.

16. You KY, Bi ZF, Liu YM, Qiu XS. Adjuvant chemotherapy for rectal cancer. Lancet Oncol. 2015;16(4):e152.

17. Valentini $\vee$. The paradox of preoperative (chemo)radiotherapy for rectal cancer. Lancet Oncol. 2015;16(2):127-8.

18. Petrelli F, Coinu A, Barni S. Adjuvant chemotherapy for rectal cancer. Lancet Oncol. 2015;16(4):e152-3.

19. Sainato A, Cernusco Luna Nunzia V, Valentini V, De Paoli A, Maurizi ER, Lupattelli M, Aristei C, Vidali C, Conti M, Galardi A, et al. No benefit of adjuvant fluorouracil Leucovorin chemotherapy after neoadjuvant chemoradiotherapy in locally advanced cancer of the rectum (LARC): long term results of a randomized trial (I-CNR-RT). Radiother Oncol. 2014;113(2):223-9.

20. Bosset JF, Calais G, Mineur L, Maingon P, Stojanovic-Rundic S, Bensadoun RJ, Bardet E, Beny A, Ollier JC, Bolla M, et al. Fluorouracil-based adjuvant chemotherapy after preoperative chemoradiotherapy in rectal cancer: long-term results of the EORTC 22921 randomised study. Lancet Oncol. 2014;15(2):184-90.

21. Maas M, Nelemans PJ, Valentini V, Crane CH, Capirci C, Rodel C, Nash GM, Kuo LJ, Glynne-Jones R, Garcia-Aguilar J, et al. Adjuvant chemotherapy in rectal cancer: defining subgroups who may benefit after neoadjuvant chemoradiation and resection: a pooled analysis of 3,313 patients. Int J Cancer. 2015:137(1):212-20.

22. Schmoll H-J, Haustermans K, Price TJ, Nordlinger B, Hofheinz R, Daisne J-F, Janssens J, Brenner B, Schmidt P, Reinel $H$ et al: Preoperative chemoradiotherapy and postoperative chemotherapy with capecitabine and oxaliplatin versus capecitabine alone in locally advanced rectal cancer: disease-free survival results at interim analysis. ASCO Meeting Abstracts 2014, 32(15_suppl):3501.

23. Rodel C, Graeven U, Fietkau R, Hohenberger W, Hothorn T, Arnold D, Hofheinz RD, Ghadimi M, Wolff HA, Lang-Welzenbach M, et al. Oxaliplatin added to fluorouracil-based preoperative chemoradiotherapy and postoperative chemotherapy of locally advanced rectal cancer (the German CAO/ARO/AIO-04 study): final results of the multicentre, open-label, randomised, phase 3 trial. Lancet Oncol. 2015;16(8):979-89.

24. Hong YS, Nam BH, Kim KP, Kim JE, Park SJ, Park YS, Park JO, Kim SY, Kim TY, $\mathrm{Kim} \mathrm{JH}$, et al. Oxaliplatin, fluorouracil, and leucovorin versus fluorouracil and leucovorin as adjuvant chemotherapy for locally advanced rectal cancer after preoperative chemoradiotherapy (ADORE): an open-label, multicentre, phase 2, randomised controlled trial. Lancet Oncol. 2014;15(11):1245-53.

25. Peeters KC, van de Velde CJ, Leer JW, Martijn H, Junggeburt JM, Kranenbarg EK, Steup WH, Wiggers T, Rutten HJ, Marijnen CA. Late side effects of shortcourse preoperative radiotherapy combined with total mesorectal excision for rectal cancer: increased bowel dysfunction in irradiated patients-a Dutch colorectal cancer group study. J Clin Oncol. 2005;23(25):6199-206.

26. Pollack J, Holm T, Cedermark B, Holmstrom B, Mellgren A. Long-term effect of preoperative radiation therapy on anorectal function. Dis Colon rectum. 2006;49(3):345-52.

27. Gervaz P, Rotholtz N, Pisano M, Kaplan E, Secic M, Coucke P, Pikarsky A, Efron J, Weiss E, Wexner S. Quantitative short-term study of anal sphincter function after chemoradiation for rectal cancer. Arch Surg. 2001;136(2):192-6.

28. Camma C, Giunta M, Fiorica F, Pagliaro L, Craxi A, Cottone M. Preoperative radiotherapy for resectable rectal cancer: a meta-analysis. JAMA. 2000;284(8): 1008-15.

29. Ansari N, Solomon MJ, Fisher RJ, Mackay J, Burmeister B, Ackland S, Heriot A Joseph D, McLachlan SA, McClure B, et al. Acute adverse events and postoperative complications in a randomized trial of preoperative shortcourse radiotherapy versus long-course Chemoradiotherapy for T3 adenocarcinoma of the rectum: trans-Tasman radiation oncology group trial (TROG 01.04). Ann Surg. 2017:265(5):882-8.

30. Marijnen CA, Kapiteijn E, van de Velde CJ, Martijn H, Steup WH, Wiggers T, Kranenbarg EK, Leer JW. Cooperative investigators of the Dutch colorectal Cancer G: acute side effects and complications after short-term preoperative radiotherapy combined with total mesorectal excision in primary rectal cancer: report of a multicenter randomized trial. J Clin Oncol. 2002;20(3):817-25.

31. Rodel C, Liersch T, Hermann RM, Arnold D, Reese T, Hipp M, Furst A, Schwella $\mathrm{N}$, Bieker M, Hellmich $\mathrm{G}$, et al. Multicenter phase II trial of chemoradiation with oxaliplatin for rectal cancer. J Clin Oncol. 2007;25(1):110-7.

32. Bakx R, Busch OR, Bemelman WA, Veldink GJ, Slors JF, van Lanschot JJ. Morbidity of temporary loop ileostomies. Dig Surg. 2004;21(4):277-81.

33. Alves $A$, Panis $Y$, Lelong $B$, Dousset $B$, Benoist $S$, Vicaut E. Randomized clinical trial of early versus delayed temporary stoma closure after proctectomy. Br J Surg. 2008;95(6):693-8.

34. Thalheimer A, Bueter M, Kortuem M, Thiede A, Meyer D. Morbidity of temporary loop ileostomy in patients with colorectal cancer. Dis Colon rectum. 2006;49(7):1011-7.

35. Sandra-Petrescu F, Herrle F, Hinke A, Rossion I, Suelberg H, Post S, Hofheinz $\mathrm{RD}$, Kienle P. CoCStom trial: study protocol for a randomised trial comparing completeness of adjuvant chemotherapy after early versus late diverting stoma closure in low anterior resection for rectal cancer. BMC Cancer. 2015;15(1):923.

\section{Submit your next manuscript to BioMed Central and we will help you at every step:}

- We accept pre-submission inquiries

- Our selector tool helps you to find the most relevant journal

- We provide round the clock customer support

- Convenient online submission

- Thorough peer review

- Inclusion in PubMed and all major indexing services

- Maximum visibility for your research

Submit your manuscript at www.biomedcentral.com/submit
) Biomed Central 